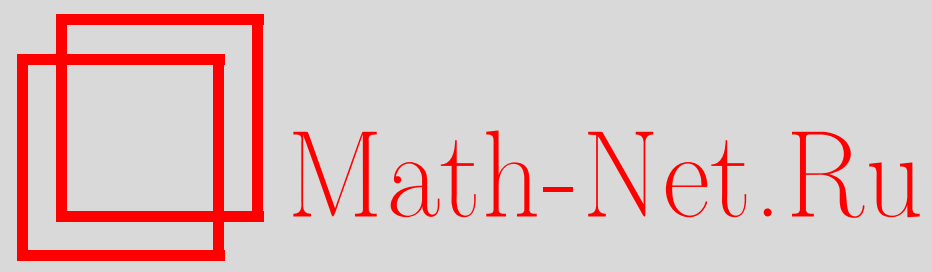

А. Ю. Маковецкий, О преобразованиях специальных спайнов и специальных полиэдров, Матем. заметки, 1999, том 65, выпуск 3, 354-361

DOI: https://doi.org/10.4213/mzm1058

Использование Общероссийского математического портала Math-Net.Ru подразумевает, что вы прочитали и согласны с пользовательским соглашением http://www . mathnet.ru/rus/agreement

Параметры загрузки:

IP : 52.6 .47 .48

26 апреля 2023 г., $14: 26: 50$

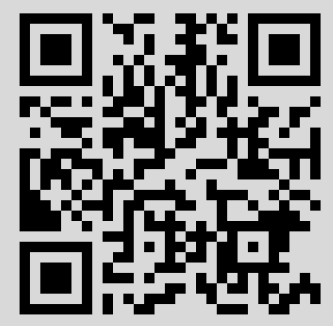




\title{
О ПРЕОБРАЗОВАНИЯХ СПЕЦИАЛЬНЫХ СПАЙНОВ И СПЕЦИАЛЬНЫХ ПОЛИЭДРОВ
}

\author{
А. Ю. Маковецкий
}

Изучаются специальные спайны 3-многообразий и специальные полиэдры. Рассматриваются специальные преобразования спайнов и полиэдров. Известно, что две триангуляции одного 3-многообразия имеют общее звездное подразделение и два разбиения Хегора одного 3-многообразия стабильно эквивалентны. Доказывается аналог этих фактов для спайнов и полиэдров. Изучаются спайны со структурой разветвленной поверхности.

Библиография: 11 названий.

Введение. В топологии трехмерных многообразий принято задавать многообразия как объединения более простых объектов. К числу таких способов задания относятся триангулящия многообразия и разбиение Хегора. Известно [1], что для двух триангуляций одного и того же многообразия справедлива теорема о существовании общего звездного подразделения. Также известно [2], что любые два разбиения Хегора одного и того же многообразия являются стабильно эквивалентными.

Кроме двух упомянутых способов задания 3-многообразий широко известен способ задания с помошью специальных спайнов, изученньй в работах Каслера [3], Матвеева [4] и Пиергаллини [5]. В классе специальных спайнов существует некоторое отношение $T$-эквивалентности, состоящее в следующем: два специальных спайна являются $T$-эквивалентными, если от одного из них можно перейти ко второму посредством применения преобразований $T_{0}^{ \pm 1}, T_{2}^{ \pm 1}$. Возникает вопрос об аналоге упомянутых вьше теорем для $T$-эквивалентных спайнов.

Этот же вопрос можно поставить и для класса специальных полиэдров.

В данной работе доказьвается теорема о существовании для любых двух $T$-эквивалентных специальных полиэдров $P$ и $Q$ такого специального полиэдра $S$, что от полиэдра $P$ к полиэдру $S$ и от полиэдра $Q$ к полиэдру $S$ можно перейти только с помощью преобразований $T_{0}$ и $T_{2}$. Также доказьвается, что для любых двух специальных спайнов $P$ и $Q$ одного и того же 3 -многообразия существует специальный спайн $S$ того же многообразия такой, что от спайна $P$ к спайну $S$ и от спайна $Q$ к спайну $S$ можно перейти только с помощью преобразований $T_{0}$ и $T_{2}$, оставаясь все время в классе специальных спайнов данного многообразия.

Тезисы данной статьи опубликованы в [6].

Работа выполнена при частичной поддержке Российского фонда фундаментальных исследований, грант № 96-01-00847, и фонда INTAS, грант № 94-921. 


\section{1. Формулировки теорем.}

ОПРеДЕЛЕнИЕ 1. Компактньй полиэдр $P$ называется специальным полиәдром, если выполняются следуюшие условия:

1) линк каждой его точки гомеоморфен одному из следуюших одномерных полиэдров:

а) окружности;

б) окружности с диаметром;

в) окружности с тремя радиусами;

2) в $P$ существует хотя бы одна точка с линком типа в);

3) любая 2-компонента, т.е. связное подмножество точек полиэдра с линком типа а), полиэдра $P$ гомеоморфна открытой двумерной клетке.

ОПРЕДЕЛЕниЕ 2. Специальный полиэдр $P$, вкладываемый в 3 -многообразие $M$ с границей, называется спеииальным спайномэтого многообразия, если многообразие $M$ коллапсируется на полиэдр $P$, т.е. многообразие $M \backslash P$ гомеоморфно прямому произведению $\partial M \times(0,1]$.

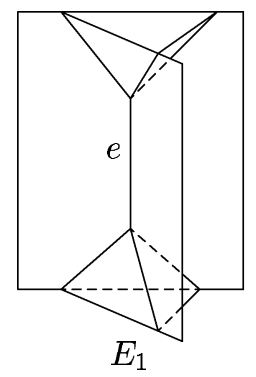

$E_{1}$
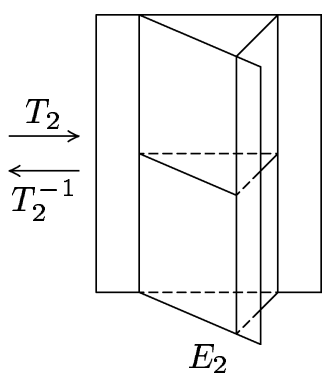

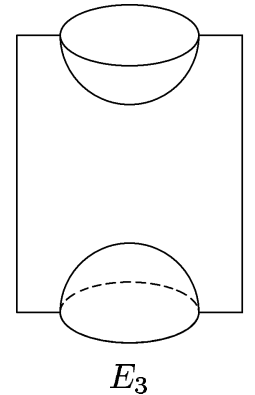

$E_{3}$

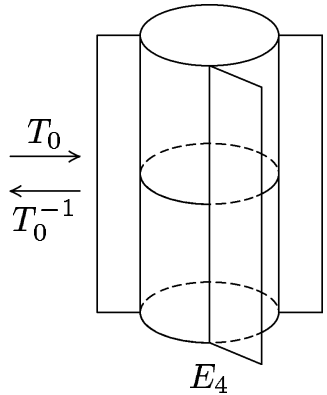

$E_{4}$

Рис. 1

Пусть $P_{1}$ - специальный полиэдр. Выберем в полиэдре $P_{1}$ ребро $e$. Рассмотрим регулярную окрестность $E_{1}$ ребра $e$ в полиэдре $P_{1}$ (см. рис. 1$)$. Пересечение окрестности $E_{1}$ с остальной частью полиэдра $P_{1}$ гомеоморфно объединению двух окружностей, соединенных тремя дугами. Рассмотрим полиэдр $E_{2}$, который представляет собой поверхность треугольной призмы вместе со средним треугольником и тремя прямоугольниками, которые присоединены к поверхности призмы вдоль ее боковых ребер.

Естественная гранища полиэдра $E_{2}$ гомеоморфна границе полиэдра $E_{1}$. Если мы заменим окрестность $E_{1}$ на окрестность $E_{2}$, то получим новьй специальный полиэдр $P_{2}$.

Замена полиэдра $P_{1}$ на полиэдр $P_{2}$ обозначается через $T_{2}$, обратная замена через $T_{2}^{-1}$.

Аналогичным образом описываются преобразования $T_{0}$ и $T_{0}^{-1}$, состоящие в замене подполиэдра $E_{3}$ на подполиэдр $E_{4}$ и наоборот (см. рис. 1$)$.

Преобразования $T_{2}$ и $T_{0}$ будем называть увеличивающими, преобразования $T_{2}^{-1}$ и $T_{0}^{-1}$ уменьиающими.

Специальные полиэдры $P$ и $Q$ будем называть $T$-эквивалентными $(P \stackrel{T}{\sim} Q)$, если от полиэдра $P$ к полиэдру $Q$ можно перейти с помощью последовательности преобразований $T_{0}^{ \pm 1}, T_{2}^{ \pm 1}$. Если от полиэдра $P$ к полиэдру $Q$ можно перейти только с помощью 
преобразований $T_{0}$ и $T_{2}\left(T_{0}^{-1}\right.$ и $\left.T_{2}^{-1}\right)$, то будем обозначать этот факт через $P \nearrow Q$ (соответственно через $P \searrow Q$ ).

Теорема 1. Если $P \stackrel{T}{\sim} Q$, то существует полиәдр $S$ такой, что $P \nearrow S \searrow Q$.

Теорема 2. Пусть $P$ и $Q$ - специальные спайны одного и того же многообразия $M^{3}$. Тогда существует специальный спайн $S$ того жсе многообразия $M^{3}$ такой, что $P \nearrow S \searrow Q$, причем все промежуточные спайны межсу $P$ и $S u$ между $S$ и $Q$ являтся специальными спайнами данного многообразия $M^{3}$.

Доказательства теорем схожи, поэтому рассмотрим доказательство теоремы 2 , после чего отметим, какие изменения надо внести в это доказательство, чтобы получить доказательство теоремы 1.

ПРЕДЛОЖЕНИЕ 1. Пусть $P_{1}, \ldots, P_{n}$ u $R$ - специальные спайны одного и того же 3-многообразия $M^{3}$, связанные друг с другом преобразованиями $T_{0}^{ \pm 1}, T_{2}^{ \pm 1}$, причем $P_{1} \searrow P_{2} \searrow \cdots \searrow P_{n} \nearrow R$, спайн $P_{i}($ спайн $R)$ получается из спайна $P_{i-1}($ из спайна $P_{n}$ соответственно) применением одного преобразования. Тогда от спайна $P_{1} \kappa$ спайну $R$ можно перейти, применяя сначала только увеличивающие, $a$ затем только уменьшающие преобразования, все время оставаясь в классе спайнов данного многообразия $M^{3}$.

Доказательству предложения 1 посвящены п. 2, 3.

\section{2. Преобразования помеченных спайнов.}

ОПРЕДЕЛЕНИЕ 3. Допустимой кривой в специальном спайне Р будем называть простую кривую $\xi \subset P$ такую, что $\xi$ трансверсально пересекается с особьм графом спайна $P$ в конечном числе точек, причем $\xi$ не содержит вершин спайна.

ОПРЕДЕЛЕНИЕ 4. Помеченным спайном будем называть пару $(P, \xi)$, где $P$ - спайн с допустимой кривой $\xi$.

Опишем элементарные преобразования помеченных спайнов.

ОПРЕДЕЛЕниЕ 5. Пусть $\xi$ - допустимая кривая в помеченном спайне $(P, \xi), I$ - единичньй отрезок, $(\xi \times I) \subset M-$ прямое произведение кривой на отрезок и $A, B-$ концы кривой $\xi$. Пусть также $(\xi \times I) \cap P=(\xi \times\{0\}) \cup(\{A, B\} \times I)$. Тогда допустимая кривая $\xi$ назьвается сталкиваемой кривой.

Пусть $(P, \xi)$ - помеченный спайн и подполиэдр $E_{1}$ является окрестностью некоторого ребра в спайне $P$, причем $E_{1} \cap \xi=\varnothing$. Заменим подполиэдр $E_{1}$ на подполиэдр $E_{2}$. Получим новый помеченньй спайн $\left(P^{\prime}, \xi\right)$. Замену помеченного спайна $(P, \xi)$ на помеченный спайн $\left(P^{\prime}, \xi\right)$ обозначим через $\widetilde{T}_{2}$. Аналогично замену подполиэдра $E_{3}$ на подполиэдр $E_{4}$ обозначим через $\widetilde{T}_{0}$.

Пусть кривая $\xi$ помеченного спайна $(P, \xi)$ в окрестности вершины $V$ спайна $P$ пересекает ровно один раз одно из ребер, примыкающих к вершине $V$. Рассмотрим кривую $\xi^{\prime}$, которая совпадает с кривой $\xi$ вне окрестности вершины $V$, а в этой окрестности два раза пересекает два разных ребра, примыкающих к $V$. Будем обозначать переход от $(P, \xi)$ к $\left(P, \xi^{\prime}\right)$ через $M_{2}$.

Обратное преобразование обозначим через $M_{2}^{-1}$. Очевидно, преобразования $M_{2}^{ \pm 1}$ сохраняют свойство кривой быть допустимой и быть сталкиваемой. 
Пусть конец кривой $\xi$ из $(P, \xi)$ в окрестности вершины $V$ спайна $P$ лежит на одном из ребер, примыкающих к вершине $V$. Рассмотрим кривую $\xi^{\prime}$, которая совпадает с кривой $\xi$ вне окрестности вершины $V$, а в этой окрестности два раза пересекает два разных ребра, примыкающих к $V$, причем одна из этих точек пересечения является концом кривой $\xi$. Будем обозначать переход от $(P, \xi)$ к $\left(P, \xi^{\prime}\right)$ также через $M_{2}$.

Преобразование $M_{0}^{ \pm 1}$ можно определить следующим образом. Пусть $\alpha$ - простая кривая в спайне $P$ такая, что $\alpha \cap S(P)=a, \alpha \cap \xi=b$, (здесь $S(P)$ - особый граф специального спайна $P, \xi$ - кривая в помеченном спайне), где $a$ и $b$ - концы кривой $\alpha$, причем точка $a$ не является вершиной спайна $P$ и точка $b \notin S(P)$.

Тогда мы можем заменить кривую $\xi$ на кривую $\xi^{\prime}$, которая совпадает с $\xi$ вне окрестности кривой $\alpha$ и $\xi^{\prime} \cap \alpha=\varnothing$, причем $\xi$ два раза пересекает ребро, содержащее точку $a$.

Преобразования $M_{0}^{ \pm 1}$ сохраняют свойство кривой быть допустимой.

Договоримся преобразование $M_{0}$ всегда выполнять так, чтобы свойство кривой быть сталкиваемой сохранялось.
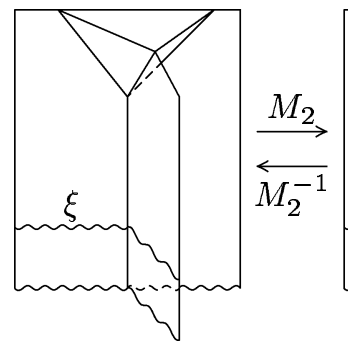
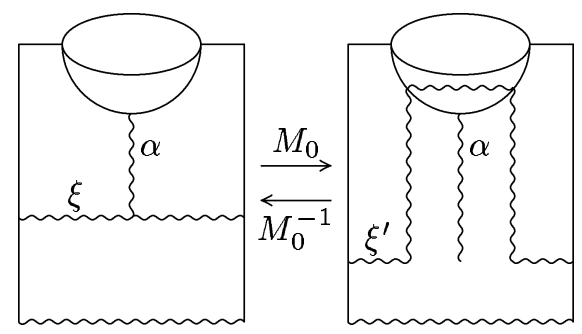

Рис. 2

Пусть $(P, \xi)$ и $(Q, \eta)$ - помеченные спайны и от $(P, \xi)$ к $(Q, \eta)$ можно перейти с помощью преобразований $\widetilde{T}_{0}, \widetilde{T}_{2}, M_{0}, M_{2}$. Этот факт будем записьвать следующим образом: $(P, \xi) \widetilde{\nearrow}(Q, \eta)$.

ЛЕмма 1. Пусть $V_{1} V_{2}$ - ребро помеченного спайна $(P, \xi)$ (или кривая $\alpha$ из определения преобразования $M_{0}$ ). Тогда с помощью преобразований $M_{2}$ и $M_{0}$ можнно получить помеченный спайн $\left(P, \xi^{\prime}\right)$ такой, что $\xi^{\prime} \cap V_{1} V_{2}=\varnothing$.

ДокАЗАТЕЛЬСТво. Пусть $\xi \cap V_{1} V_{2}=p_{1}, \ldots, p_{k}$ и между $V_{1}$ и $p_{1}$ не лежит ни одной точки $p_{i}$. Тогда применим к кривой $\xi$ преобразование $M_{2}$. Число точек пересечения получившейся кривой и ребра $V_{1} V_{2}$ уменьшится. Случай, когда $V_{1} V_{2}-$ кривая, рассматривается аналогично.

Лемма 2. Пусть $(P, \xi)$ - помеченный спайн и пусть $P \nearrow Q$. Тогда в спайне $Q$ существует допустимая кривая $\eta$ такая, ито $(P, \xi) \widetilde{\nearrow}(P, \eta)$.

ДокАЗАТЕЛЬСТво. Если в последовательности преобразований $P \nearrow Q$ мы имеем нуль преобразований, то утверждение вьполняется.

Индукционное предположение. Если последовательность состоит из $k$ преобразований, то утверждение леммы 2 выполняется.

Рассмотрим индукционный шаг. Пусть последовательность состоит из $k+1$ преобразования. 
Предположим, что первое преобразование, применяемое к спайну $P$, есть $T_{2}$ (случай $T_{0}$ аналогичен). Обозначим через $P^{\prime}$ спайн, полученньй из спайна $P$ преобразованием $T_{2}$. Рассмотрим окрестность двух вершин $V_{1}$ и $V_{2}$ спайна $P$, к которым применяется преобразование $T_{2}$.

По лемме 1 с помощью преобразований $M_{2}$ можно получить помеченньй спайн $\left(P, \xi^{\prime}\right)$ такой, что кривая $\xi^{\prime}$ лежит в стороне от ребра $V_{1} V_{2}$. Применим преобразование $\widetilde{T}_{2}$ вдоль ребра $V_{1} V_{2}$. Полученньй спайн $P^{\prime}$ будет помеченным спайном с кривой $\xi^{\prime}$. Спайн $P^{\prime}$ связан со спайном $Q$ посредством $k$ преобразований. По предположению индукции кривая $\eta$ в помеченном спайне $(Q, \eta)$ будет искомой кривой.

3. Вздутия помеченных спайнов. Пусть $(P, \xi)$ - помеченньй спайн, причем допустимая кривая $\xi$ является сталкиваемой.

Пусть $\xi \times I-$ прямое произведение кривой на единичньй отрезок такое, что $(\xi \times I) \subset P$, $(\xi \times I) \cap V(P)=\varnothing$, где $V(P)$ - множество вершин спайна $P$, и любая точка пересечения кривой $\xi \times\{1\}$ с особым графом $S(P)$ спайна $P$ совпадает с точкой $\mathrm{cr}_{i}(\xi) \times\{1\}$, где $\mathrm{cr}_{i}(\xi)$ - некоторая точка пересечения кривой $\xi$ с графом $S(P)$. Тогда про кривую $\xi \times\{1\}$ будем говорить, что она параллельна кривой $\xi$.

Рассмотрим кривую $\xi^{\prime}$ параллельную кривой $\xi$. Обозначим конщы кривых $\xi$ и $\xi^{\prime}$ через $A, B$ и $A^{\prime}, B^{\prime}$. Соединим точки $A$ и $A^{\prime}$ кривой $\psi$, точки $B$ и $B^{\prime}$ кривой $\psi^{\prime}$ так, как показано на рис. 3 .

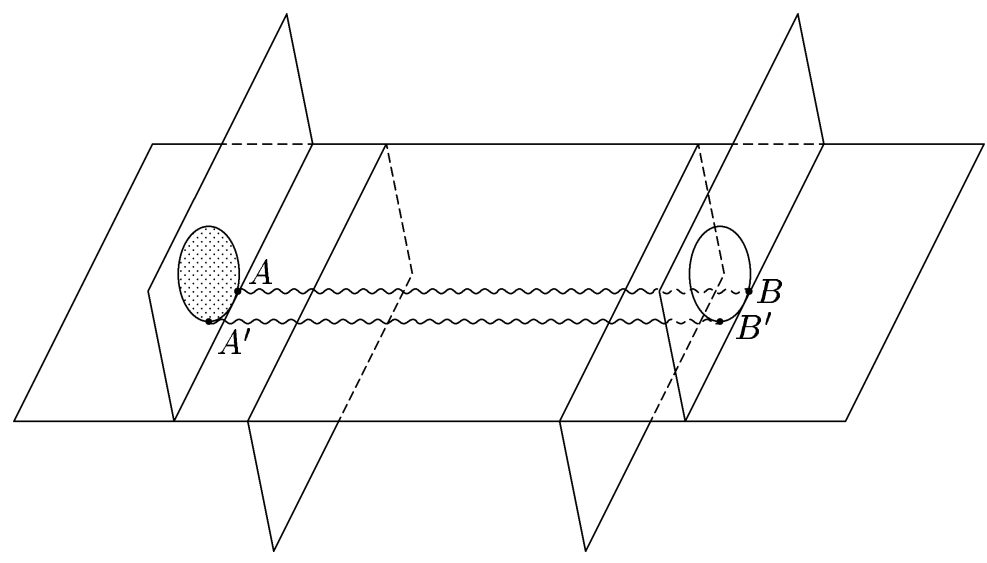

Рис. 3

Объединение четырех кривых $\xi, \xi^{\prime}, \psi$ и $\psi^{\prime}$ гомеоморфно окружности; приклеим к этой окружности диск $D^{2}$. Проколем диск, границей которого является объединение отрезка $A A^{\prime}$ и кривой $\psi$ (на рис. 3 этот диск заштрихован). Получившийся спайн обозначим через $\operatorname{sw}(P, \xi)$ и будем назьвать вздутием спайна $P$ вдоль кривой $\xi$.

Лемма 3. Пусть $(P, \xi) \widetilde{\nearrow}(Q, \eta) . \quad$ Тогдa $\operatorname{sw}(P, \xi) \nearrow \operatorname{sw}(Q, \eta)$.

ДокАЗАТЕЛЬСтво. Если в последовательности $(P, \xi) \nearrow(Q, \eta)$ мы имеем 0 преобразований, то утверждение вьполняется. Предположим, что если последовательность состоит из $k$ преобразований, то утверждение также выполняется.

Индукционный шаг. Рассмотрим последовательность из $k+1$ преобразований. Если первое увеличивающее преобразование, примененное к спайну $P$, есть $\widetilde{T}_{0}$ или $\widetilde{T}_{2}$, то утверждение леммы 3 выполняется. 
Допустим, что первое увеличивающее преобразование, применяемое к помеченному спайну $(P, \xi)$, есть $T_{2}$ (случай преобразования $T_{0}$ рассматривается аналогично). Тогда по лемме 1 можно добиться того, чтобы допустимая кривая $\xi$ не пересекалась с окрестностью ребра $E$, вдоль которого производится преобразование $T_{2}$. Проследим, что происходит со вздутием спайна $P$ вдоль кривой $\xi$ при применении к кривой $\xi$ преобразований $M_{0}, M_{2}$.

Возможны три случая:

1 ) на ребре $E$ не лежит ни начало $A$, ни конец $B$ кривой $\xi$;

2 ) на ребре $E$ лежит конец кривой $\xi$;

$3)$ на ребре $E$ лежит начало $A$ кривой $\xi$.

Рассмотрим случай 3) как наиболее сложный.
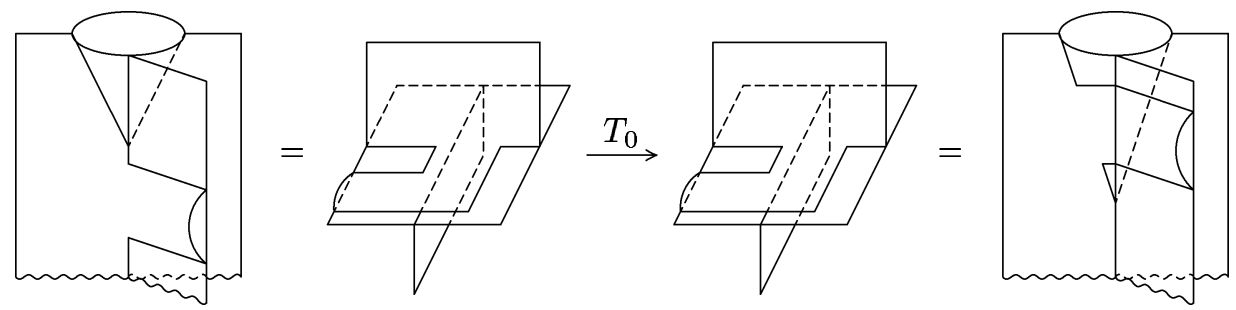

Рис. 4

Переход от одного спайна со вздутием к другому осушествляется с помощью одного преобразования $T_{0}$, как показано на рис. 4.

Рассмотренные случаи доказьвают лемму 3.

Лемма 4. Пусть $(P, \xi)-$ помеченный спайн. Тогда $(P, \xi) \nearrow \operatorname{sw}(P, \xi)$.

ДокАЗАТЕЛЬСтво. Если число точек пересечения кривой $\xi$ равно 2 , то от $(P, \xi)$ $\mathrm{k} \mathrm{sw}(P, \xi)$ можно перейти с помощью одного преобразования $T_{0}$. Если число таких точек равно $n$, то требуется $n-1$ преобразований $T_{0}$.

ДОКАЗАТЕЛЬСТВО ПРЕДЛОЖЕНИЯ 1 . Если переход от спайна $P_{n}$ к спайну $R$ осуществляется с помощью преобразования $T_{2}$, то на спайне $P_{n}$ в качестве допустимой кривой $\xi$ возьмем кривую параллельную ребру, задающему преобразование $T_{2}$. Если переход осуществляется с помощью преобразования $T_{0}$, то возьмем в качестве допустимой кривой $\xi$ кривую, задающую это преобразование. По лемме 2 существует кривая $\eta$ на $P_{1}$ такая, что $\left(P_{1}, \eta\right) \searrow\left(P_{n}, \xi\right)$. По лемме 4 для допустимой пары $(P, \xi)$ мы имеем переход $\left(P_{1}, \eta\right) \nearrow \mathrm{sw}\left(P_{1}, \eta\right)$.

По лемме 3 существует переход $\mathrm{sw}\left(P_{1}, \eta\right) \searrow \mathrm{sw}\left(P_{n}, \xi\right)$. Если $P_{n}$ и $Q$ связаны преобразованием $T_{0}, \operatorname{Tosw}\left(P_{n}, \xi\right)=Q$. Если $P_{n}$ и $Q$ связаны преобразованием $T_{2}$, то от $\operatorname{sw}\left(P_{n}, \xi\right)$ к $Q$ можно перейти с помощю одного преобразования $T_{2}$ так, как показано на рис. 5 .

Тем самым предложение 1 доказано.

4. Применение полученных результатов. Покажем, как из предложения 1 можно вывести теорему 2. Рассмотрим спайны $P$ и $S$ одного и того же многообразия $\mathrm{M}^{3}$, связанные последовательностью преобразований $T_{0}^{ \pm 1}, T_{2}^{ \pm 1}$. K спайну $P$ применяется сначала некоторое число увеличивающих преобразований. Обозначим это число через $I_{1}$. 

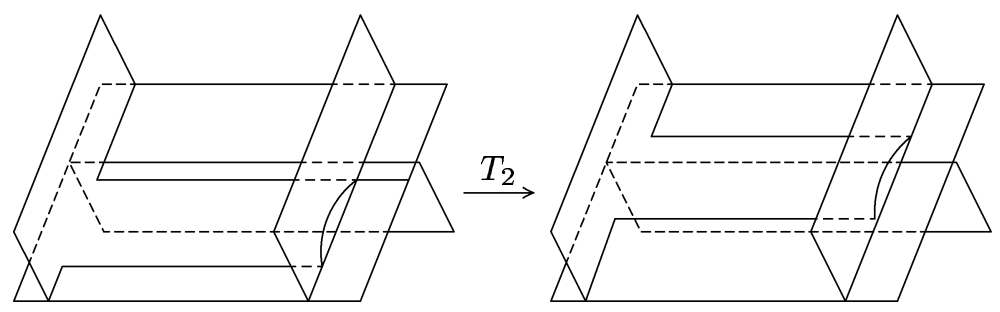

Рис. 5

Затем применяется некоторое число уменьшающих преобразований, после чего применяется $I_{2}$ увеличивающих преобразований и т.д., $I_{k}$ увеличивающих преобразований. Число $I_{1}$ может быть равно 0. Назовем число $W=\sum_{i=2}^{k} I_{i}$ параметром последовательности. Применяя предложение 1 к последовательности преобразований, состоящей из некоторого числа уменьшающих преобразований и следующего за ними первым увеличивающего преобразования, дающего вклад в число $I_{2}$, можно получить новую последовательность с параметром $W-1$, так как число $I_{2}$ уменьшится на 1 . Последовательно применяя предложение 1 , получим последовательность с параметром $W=0$, т.е. последовательность нужного вида. Теорема 2 доказана.

Опишем изменения, которые нужно внести в доказательство теоремы 2 , чтобы получить доказательство теоремы 1.

Для допустимой кривой в полиэдре не определяется свойство сталкиваемости.

В случае полиэдра с допустимой кривой вздутие определяется как результат применения $n-1$ преобразований $T_{0}$, где $n$ - число точек пересечения допустимой кривой с ребрами полиэдра. В остальном доказательство теоремы 1 повторяет доказательство теоремы 2 .

\section{5. Дополнительные структуры на специальных спайнах 3-многообразий.} Вопросы, связанные с изучением разветвленных спайнов, были, в частности, рассмотрены в работах Гиллмана и Ролфсена [7], [8] и в работах Ишии [9]-[11].

Типичные окрестность ребра и окрестность вершины разветвленного спайна показаны на рис. 6.
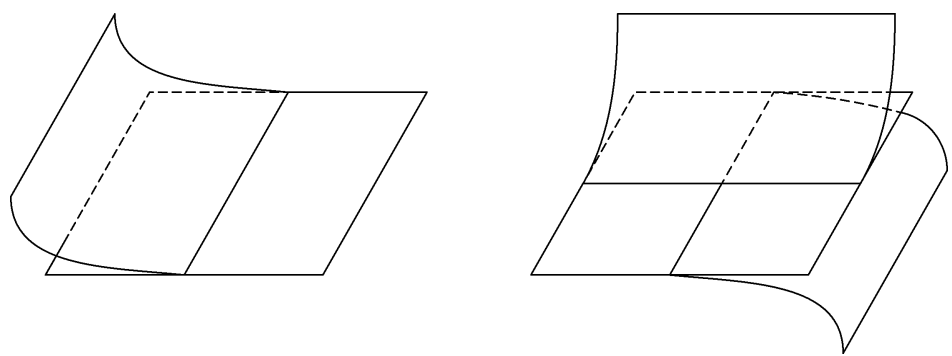

Рис. 6

Будем называть крыльями в окрестности ребра (вершины) части клеток, инцидентных данному ребру (вершине), попадающие в данную окрестность. Таким образом, в окрестности каждого ребра (вершины) имеется три крыла (шесть крыльев). Будем на- 
зьвать два крыла в окрестности вершины противоположными, если они пересекаются только в одной точке (т.е. в соответствующей вершине).

Можно определить разветвленньй спайн с помощью эквивалентного следующего определения.

ОПРЕДЕЛЕНИЕ 6. Разветвленным спайном называется спайн, у которого каждому ребру сопоставлен отрезок, один конец которого лежит на данном ребре, а другой конец вместе со всеми остальньми точками отрезка лежит на одном из трех крыльев, примыкающих к данному ребру, причем в окрестности каждой вершины должно вьполнятся следующее условие: два отрезка из четырех должны лежать на двух противоположных крыльях, а два оставшихся отрезка должны лежать на одном и том же крыле (других отрезков на этом крыле лежать не должно).

ТЕОРемА 3. Пусть $P$ - разветвленный спайн и спайн $S$ получается из спайна $P$ применением к $P$ увеличивающего преобразования $T_{2}$ или $T_{0}$. Тогда спайн $S$ также является разветвленным спайном.

ДокАЗАТЕЛЬСТвО. С точностью до гомеоморфизма окрестности двух соседних вершин разветвленного спайна на себя существует шесть различных расстановок отрезков в этой окрестности. Применяя к этой окрестности преобразование $T_{2}$ для каждого из шести случаев, можно убедиться, что на полученном спайне $S$ существует расстановка отрезков, задающая разветвленную структуру на спайне. Подобное рассуждение проходит и для преобразования $T_{0}$.

Автор благодарен научному руководителю С. В. Матвееву за помощь в работе над статьей.

\section{СПИСОК ЦИТИРОВАННОЙ ЛИТЕРАТУРЫ}

[1] Alexander J. W. The combinatorial theory of complexes // Ann. of Math. (2). 1930. V. 31. P. 294-322.

[2] Singer J. Three-dimensional manifolds and their Heegaard diagrams // Trans. Amer. Math. Soc. 1933. V. 35. P. 88-111.

[3] Casler B. G. An embedding theorem for connected 3-manifolds with boundary // Proc. Amer. Math. Soc. 1965. V. 16. P. 559-566.

[4] Matveev S. V. Transformations of special spines and Zeeman conjecture // Math. USSR Izv. 1988. V. 31. P. 423-434.

[5] Piergallini R. Standard moves for standard polyhedra and spines // Rend. Circ. Mat. Palermo (2). 1988. V. 37. № 18. P. 391-414.

[6] Macovetsky A. Transformations on special spines of 3-manifold and branched surfaces // Knots'96 Conference. Workshop Report for 5th MSJ International Research Institute Knot Theory. Tokyo, 1996. P. 126-127.

[7] Gillman D., Rolfsen D. The Zeeman conjecture for standard spines is equivalent to the Poincaré conjecture // Topology. 1983. V. 22. P. 315-323.

[8] Gillman D., Rolfsen D. Three-manifolds embedded in small 3-complexes // Internat. J. Math. 1992. V. 3. P. 179-183.

[9] Ishii I. Flows and spines // Tokyo J.Math. 1986. V. 9. P. 505-525.

[10] Ishii I. Combinatirial construction of a non-singular flow on 3-manifold // Kobe J. Math. 1986. V. 3. P. 201-208.

[11] Ishii I. Moves for flow spines and topological invariants of 3-manifold // Tokyo J. Math. 1992. V. 15. P. 297-312. 